\title{
New Approach of Electromagnetic Fields of the Lightning Discharge
}

\author{
${ }^{1}$ Dib Djalel, ${ }^{2}$ Mordjaoui Mourad and ${ }^{3}$ Hocine Labar \\ ${ }^{1}$ Department of Electrical Engineering, \\ Laboratory of Electrical Engineering LABGET, University of Tebessa, 12002, Algeria \\ ${ }^{2}$ Department of Electrical Engineering, University of Skikda, Skikda, Algeria \\ ${ }^{3}$ Department of Electrical Engineering, University of Annaba, Annaba, Algeria
}

Received 2012-08-12; Revised 2013-01-22; Accepted 2014-01-18

\begin{abstract}
Despite the significant developments in the protection means of electrical and electronic systems against the lightning and its effects. With its unpredictability and aggress if character, the lightning is the most dangerous phenomenon for electrical systems, which requires more interest and greater effort by researchers and designers means of protection. We present in this study a new analytic model of transient electromagnetic fields radiated by the lightning channel. To better estimate our new model, we developed a program 'SIMLIGHTINING' with Matlab to simulated and approved all mathematic components proposed in this study. The results obtained were compared with other simulations and experiment already published have given a very appreciable similar and affinity.
\end{abstract}

Keywords:Lightning, Radiation, Electromagnetic Fields, Return Current Stroke, SIMLIGHTNING, Modeling, Simulation

\section{INTRODUCTION}

Knowledge of the characteristics of electric and magnetic fields produced by lightning discharges is needed for studying the effects of the potentially deleterious coupling of lightning fields to various electric systems. Sensitive electronic circuits are particularly vulnerable to such effects. The computation of lightning electric and magnetic fields requires the use of a model that specifies current as a function of time at all points along the radiating lightning channel. The computed fields can be used as an input to electromagnetic coupling models, the latter, in turn, being used for the calculation of lightning induced voltages and currents in various circuits and systems. For the purpose of simulating and interpreting the effects of a lightning flash to earth, it is helpful to have a simple mathematical expression describing the spatial-temporal distribution of lightning current along the channel and its associated electromagnetic fields. In this study, we will briefly describe some established approaches to the electromagnetic fields associated with the return stroke phase of a lightning discharge. There is no intention to present an exhaustive literature survey and, in what follows, only those models of interest for engineering applications due to their relative simplicity, with small number of unknowns, will be treated.

The evaluation of electromagnetic effects associated with a lightning return stroke process generally includes automatically the following points:

- Characterization and representation of the return stroke channel base current;

- Specification of the spatial-temporal distribution of the return-stroke current along the channel (using return-stroke models)

- Calculation of radiated electromagnetic fields

- Modeling the coupling of electromagnetic fields to electrical systems. This last part is not covered in

Corresponding Author: Dib Djalel, Department of Electrical Engineering, Laboratory of Electrical Engineering LABGET, University of Tebessa, 12002, Algeria 
this thesis and the reader can find exhaustive analyses in the literature

We based on the famous analytical formulation of $\mathrm{M}$. Uman and all Equation (1 to 3) published in 1975 and the current in the lightning Canal model by the engineers of MTLE Nucci and Rachidi (1989) and Cooray (1994), our analytical and numerical development with theoretical tools such as the parity symmetric integration, the Numerical integration of Trapez and method of the difference operator of first order has led to expressions only in the time domain substituting the other variable parameters witch render the initial expression complicated. To better estimate our new model of the electromagnetic field, we developed a program module under Matlab named SIMLIGHTINING for simulation and approving the proposed model

\section{ELECTROMAGNETIC FIELD PRODUCED BY THE LIGHTNING DISCHARGE}

Due to the current that flows in the lightning channel, an electromagnetic field is radiated from the lightning. The visible part of the spectrum of this field represents only a small part of the whole electromagnetic energy, dissipated by the lightning stroke. The instantaneous energy released from the stroke is so big that, even at the distance of 100 $\mathrm{km}$, the amplitude of the electric field pulse wave amounts to several V/m. Figure 1 shows a schematic representation of the lightning channel's assumed geometry and indicates also the observation point $\mathrm{P}$ where the fields will be calculated. The cylindrical coordinate system is adopted to represent the fields in this geometry.

Several authors have treated the complete problem of the electromagnetic radiation of a dipole over a finitely conducting half-space by determining the solution of Maxwell's equations for both media in accordance with the boundary conditions on the airground interface. The resulting equations are obtained in the frequency domain and are in terms of slowly converging integrals (Sommerfeld integrals). The problem is greatly simplified if one assumes a perfectly-conducting ground. In that case, the components of the electric and magnetic fields at the location $\mathrm{P}(\mathrm{r}, \phi \square, \mathrm{z})$ produced by a short vertical section of infinitesimal channel dz' at height $z^{\prime}$ carrying a timevarying current $i\left(z^{\prime}, t\right)$ can be computed in the time domain using the following relations (Heidler, 1985; Nucci and Rachidi, 1989; Rachidi et al., 1998).

\subsection{Playback on the Analytic Model of Electromagnetic Fields}

\subsubsection{Horizontal Electric Field}

$$
\begin{aligned}
& \mathrm{E}_{\mathrm{r}}(\mathrm{r}, \mathrm{z}, \mathrm{t})=\frac{1}{4 \pi \varepsilon_{\mathrm{o}}}\left[\int_{-\mathrm{H}}^{\mathrm{H}} \frac{3 \mathrm{r}\left(\mathrm{z}-\mathrm{z}^{\prime}\right)}{\mathrm{R}^{5}} \int_{0}^{\mathrm{t}} \mathrm{i}\left(\mathrm{z}^{\prime}, \tau-\mathrm{R} / \mathrm{c}\right) \mathrm{d} \tau \mathrm{dz} \mathrm{z}^{\prime}\right. \\
& +\int_{-\mathrm{H}}^{\mathrm{H}} \frac{3 \mathrm{r}\left(\mathrm{z}-\mathrm{z}^{\prime}\right)}{\mathrm{cR}^{4}} \mathrm{i}\left(\mathrm{z}^{\prime}, \mathrm{t}-\mathrm{R} / \mathrm{c}\right) \mathrm{dz} \\
& \left.+\int_{-\mathrm{H}}^{\mathrm{H}} \frac{\mathrm{r}^{2}}{\mathrm{c}^{2} \mathrm{R}^{3}} \frac{\partial \mathrm{i}\left(\mathrm{z}^{\prime}, \mathrm{t}-\mathrm{R} / \mathrm{c}\right)}{\partial \mathrm{t}} \mathrm{dz} \mathrm{z}^{\prime}\right]
\end{aligned}
$$

\subsubsection{Vertical Electric Field}

$$
\begin{aligned}
\mathrm{E}_{\mathrm{z}}(\mathrm{r}, \mathrm{z}, \mathrm{t}) & =\frac{1}{4 \pi \varepsilon_{\mathrm{o}}}\left[\int_{-\mathrm{H}}^{\mathrm{H}} \frac{2\left(\mathrm{z}-\mathrm{z}^{\prime}\right)^{2}-\mathrm{r}^{2}}{\mathrm{R}^{5}} \int_{0}^{\mathrm{t}} \mathrm{i}\left(\mathrm{z}^{\prime}, \tau-\mathrm{R} / \mathrm{c}\right) \mathrm{d} \tau \mathrm{d} \mathrm{z}^{\prime}+\right. \\
& +\int_{-\mathrm{H}}^{\mathrm{H}} \frac{2\left(\mathrm{z}-\mathrm{z}^{\prime}\right)^{2}-\mathrm{r}^{2}}{\mathrm{c} \mathrm{R}^{4}} \mathrm{i}\left(\mathrm{z}^{\prime}, \mathrm{t}-\mathrm{R} / \mathrm{c}\right) \mathrm{dz} \mathrm{z}^{\prime} \\
& \left.-\int_{-\mathrm{H}}^{\mathrm{H}} \frac{\mathrm{r}\left(\mathrm{z}-\mathrm{z}^{\prime}\right)}{\mathrm{c}^{2} \mathrm{R}^{3}} \frac{\partial \mathrm{i}\left(\mathrm{z}^{\prime}, \mathrm{t}-\mathrm{R} / \mathrm{c}\right)}{\partial \mathrm{t}} \mathrm{dz} \mathrm{z}^{\prime}\right]
\end{aligned}
$$

\subsubsection{Azimuthal Magnetic Field}

$$
\begin{aligned}
& \mathrm{B}_{\phi}(\mathrm{r}, \mathrm{z}, \mathrm{t})=\frac{\mu_{\mathrm{o}}}{4 \pi}\left[\int_{-\mathrm{H}}^{\mathrm{H}} \frac{\mathrm{r}}{\mathrm{R}^{3}} \mathrm{i}\left(\mathrm{z}^{\prime}, \mathrm{t}-\mathrm{R} / \mathrm{c}\right) \mathrm{dz} \mathrm{z}^{\prime}\right. \\
& \left.+\int_{-\mathrm{H}}^{\mathrm{H}} \frac{\mathrm{r}}{\mathrm{cR}^{2}} \frac{\partial \mathrm{i}\left(\mathrm{z}^{\prime}, \mathrm{t}-\mathrm{R} / \mathrm{c}\right)}{\partial \mathrm{t}} \mathrm{dz} \mathrm{z}^{\prime}\right]
\end{aligned}
$$

and:

$$
\mathrm{R}=\sqrt{\left(\mathrm{z}-\mathrm{z}^{\prime}\right)^{2}+\mathrm{r}^{2}} ; \mathrm{H}=\mathrm{v}\left(\mathrm{t}-\frac{\mathrm{R}}{\mathrm{c}}\right)
$$

Where:

$\mathrm{i}\left(\mathrm{z}^{\prime}, \mathrm{t}\right)=$ The current carried by the dz' dipole at time $\mathrm{t}$;

$\varepsilon_{0}=$ The permittivity of the vacuum

$\mu_{\mathrm{o}} \quad=$ The permeability of the vacuum

$\mathrm{c}=$ The speed of light

$\mathrm{R}=$ The distance from the dipole to the observation point

$r=$ The horizontal distance between the channel and the observation point

In Equation ( 1 and 2), the terms containing the integral of the current (charge transferred through dz') are called "electrostatic fields" and because of their $1 / \mathrm{r} 3$ distance dependence, they are the dominant field component close to the source. The terms containing the derivative of the current are called "radiation fields" and, due to their $1 / \mathrm{r}$ distance dependence, they are the dominant component far from the source. 


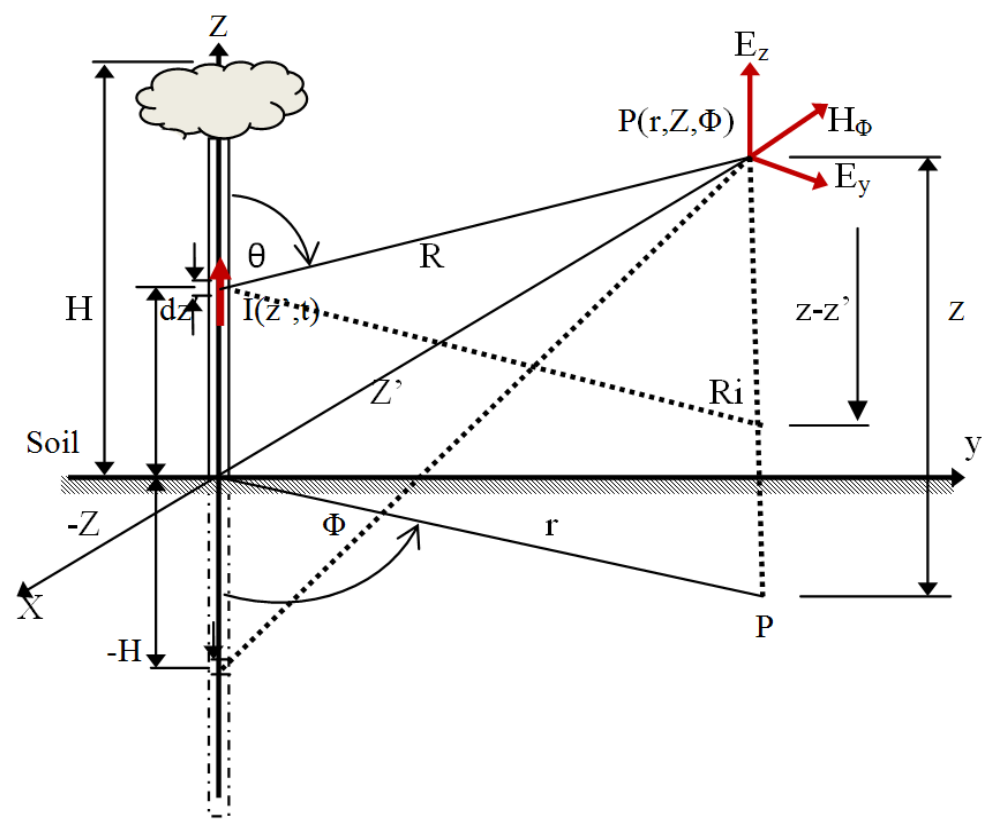

Fig. 1. Geometrical model used in calculating electromagnetic fields

The terms containing the current are called "induction fields". In Equation (3), the first term is called "induction magnetostatic field" and is the dominant field component near the source and the second term is called "radiation field" and is the dominant field component at far distances from the source.

In these equations the presence of the perfectly conducting ground is taken into account by replacing the ground by an equivalent image as shown in Fig. 1. The total fields produced by the return stroke current at the observation point are obtained by integration of the previous equations along the channel and its image.

For distance ranges beyond several kilometers, the propagation over a ground of finite conductivity results in a noticeable attenuation of high frequency components of the fields (Dib et al., 2007; Cooray, 2001).

At distances from the lightning channel not exceeding about one kilometer, the vertical component of the electric field and the azimuthal magnetic field can be calculated with reasonable approximation assuming the ground as a perfect conductor (Cooray, 1994; Rachidi et al., 1998). However, the horizontal (radial) component of the electric field radiated by lightning is more affected by a finite ground conductivity. Although at some meters above ground its intensity is much smaller than that of the vertical component, within the context of certain field-to-transmission line coupling models (Cooray, 2003; Diendorfer and Uman, 1990;
Guerrieri et al., 1996; Agrawal et al., 1980), the horizontal electric field plays an important role and thus, its calculation requires the use of the rigorous expressions or at least reasonable approximations of those. Of the many approximations proposed in the literature, the Cooray-Rubinstein formula (Cooray, 1994; 2001; Guerrieri et al., 1998) represent an efficient tool and it allows the computation of the horizontal electric field above a finitely-conducting ground with reasonable accuracy.

The calculation of the electromagnetic field requires the knowledge of the spatial-temporal distribution of the current along the channel, $i\left(z^{\prime}, t\right)$. This distribution is specified using a return stroke current model. In the following table we can summarized the existing models on the space-temporal distribution of the current return stroke of the lightning.

\subsection{Current at the Base of the Channel Lightning}

This is the only measurable characteristic, it is an important contribution in the spatio-temporal modeling of arc current back along the lightning channel. Different analytical expressions can be used to simulate the lightning current of these, found in the double exponential function, used by a number of authors who have the advantage of having analytical Fourier transforms, which allows a direct analysis in the frequency domain (Dib et al., 2007). 


\section{Fonction 1 Equation (4):}

$i(0, t)=I_{01} \cdot\left(e^{-\alpha t}-e^{-\beta t}\right)+I_{02} \cdot\left(e^{-\gamma t}-e^{-\delta t}\right)$

$\mathrm{I}_{\mathrm{o} 1}, \mathrm{I}_{\mathrm{o} 2}, \alpha, \beta, \gamma$ and $\delta$ : are the parameters which determine the exponential wave form (Guerrieri et al., 1996).

In more recent publications concerning the simulation of the subsequent return stroke, a more appropriate analytical expression was proposed. The expression consists of the sum of two socalled Heidler (1985) functions.

\section{Fonction 2:}

$$
\begin{aligned}
& i(0, t)=\frac{I_{01}}{\eta_{1}} \frac{\left(t / \tau_{11}\right)^{n}}{1+\left(t / \tau_{11}\right)^{n}} \exp \left(-t / \tau_{12}\right) \\
& +\frac{I_{02}}{\eta_{2}} \frac{\left(t / \tau_{21}\right)^{n}}{1+\left(t / \tau_{21}\right)^{n}} \exp \left(-t / \tau_{22}\right)
\end{aligned}
$$

$$
\eta_{\mathrm{i}}=\exp \left[-\left(\frac{\tau_{\mathrm{i} 1}}{\left.\tau_{\mathrm{i} 2}\right)}\right)\left(\mathrm{n} \frac{\tau_{\mathrm{i} 2}}{\tau \mathrm{i}_{1}}\right)^{\frac{1}{\mathrm{n}}}\right]
$$

And:

$\mathrm{i}=1,2$

$\mathrm{I}_{0 \mathrm{i}}=$ Amplitude parameter of the channel base current

$\tau_{\mathrm{i} 1}=$ Front time constant

$\tau_{\mathrm{i} 2}=$ Decay time constant

$\eta=$ Amplitude correction factor

$\mathrm{n}=$ Exponent $(2 \ldots 10)$

Parameters of Equation (5) for the typical current waveform at the base of a lightning are given in Table 1.

The current waveform at the base of lightning channel calculated with parameters of case 1 is represented in Fig. 2. On this Figure are also plotted the double exponential function of Heidler which compose the Equation (5). It should be and Fig. 3 that the analytical expressions for the current waveforms computed by Verbanov (2004) with the parameters of Table 1 case 1 . We can observe a great similarity between our simulation code SIMLIGHTNING, Verbanov (2004) and that measured experimentally and published by (Heidler and Hopf, 1994; Nucci and Rachidi, 1989; Rachidi et al., 1992).

The function (5) is preferred to the double exponential function (4), because it allows one to easily change return stroke current amplitude, maximum current derivative and the electrical charge transferred to the ground by simply exchanging $\mathrm{I}_{0 \mathrm{i}}, \tau_{\mathrm{i} 1}$ and $\tau_{\mathrm{i} 2}$ respectively. Equation (5) also has a time derivative equal to zero at $\mathrm{t}=0$, which is in agreement with the measured wave shapes of return stroke currents.

\subsection{The MTLE, Modified Transmission Line Model}

Published by Guerrieri et al. (1998), the model MTLE in Equation (6) corrects the defects of the TL model while keeping its simplicity by allowing an easy use in the coupling computation, based on this formulation of the space-temporal distribution along the channel of the current $i\left(z^{\prime}, t\right)$, defined by:

$$
\begin{aligned}
i\left(z^{\prime}, t\right) & =i\left(0, t-z^{\prime} / v\right) \exp \left(-z^{\prime} / \lambda\right) & z^{\prime} \leq v t \\
i\left(z^{\prime}, t\right) & =0 & z^{\prime}>v t
\end{aligned}
$$

Where:

$$
\begin{aligned}
\lambda= & \text { The decay constant which allows the current to } \\
& \text { reduce its amplitude with height } \\
\mathrm{v}= & \text { The speed of propagation of the return stroke } \\
& \text { wave front }
\end{aligned}
$$

\section{DEVELOPMENT OF A NEW MODEL OF ELECTROMAGNETIC FIELDS RADIATED BY LIGHTNING}

The development of a new model of electromagnetic fields requires an adequate choice of the transient current propagating in the lightning Canal and therefore also that at the base of the lightning Canal. Through our reading in published research, the formulation that have demonstrated their effectiveness and validated by several authors are those of MTLE by F. Rachidi crossing the channel from the ground to the cloud and Heidler model to the current the base of the channel.

The theorem of parity symmetric integrals:

$$
\int_{-H}^{H} f(z) d z=\int_{0}^{H} f(z) d z=\int_{0}^{H} f(-z) d z
$$

We set Equation (7-9):

$$
\begin{aligned}
& \mathrm{t}_{0}=0, \mathrm{t}_{\mathrm{j}+1}-\mathrm{t}_{\mathrm{j}}=\mathrm{k}=\frac{\mathrm{t}}{\mathrm{n}} \forall \mathrm{j}=1, \ldots . . \mathrm{n}-1, \mathrm{t}_{\mathrm{n}}=\mathrm{t} \text { avec } 0<\mathrm{k}<<1 \\
& \mathrm{R}=\sqrt{\mathrm{r}^{2}+\left(\mathrm{z}+\mathrm{z}^{\prime}\right)^{2}} \text { ou } \tilde{\mathrm{R}}=\sqrt{\mathrm{r}^{2}+\left(\mathrm{H}+\mathrm{Z}^{\prime}\right)^{2}} \\
& \mathrm{R}_{\mathrm{m}}=\sqrt{\mathrm{r}^{2}+(\mathrm{z}+\mathrm{mh})^{2}} \text { ou } \tilde{\mathrm{R}}_{\mathrm{m}}=\sqrt{\mathrm{r}^{2}+(\mathrm{H}+\mathrm{mh})^{2}} \\
& \mathrm{i}\left(\mathrm{z}^{\prime}, \mathrm{t}-\frac{\mathrm{R}}{\mathrm{C}}\right)=\mathrm{I}_{\mathrm{o}}\left\{\begin{array}{l}
\exp -\alpha\left(\mathrm{t}-\frac{\mathrm{R}}{\mathrm{c}}-\frac{\mathrm{z}^{\prime}}{\mathrm{v}}\right)- \\
\exp -\beta\left(\mathrm{t}-\frac{\mathrm{R}}{\mathrm{c}}-\frac{\mathrm{z}^{\prime}}{\mathrm{v}}\right)
\end{array}\right\} \exp \left(\frac{-\mathrm{z}^{\prime}}{\lambda}\right)
\end{aligned}
$$


Dib Djalel et al. / American Journal of Engineering and Applied Science 6 (4): 369-383, 2013

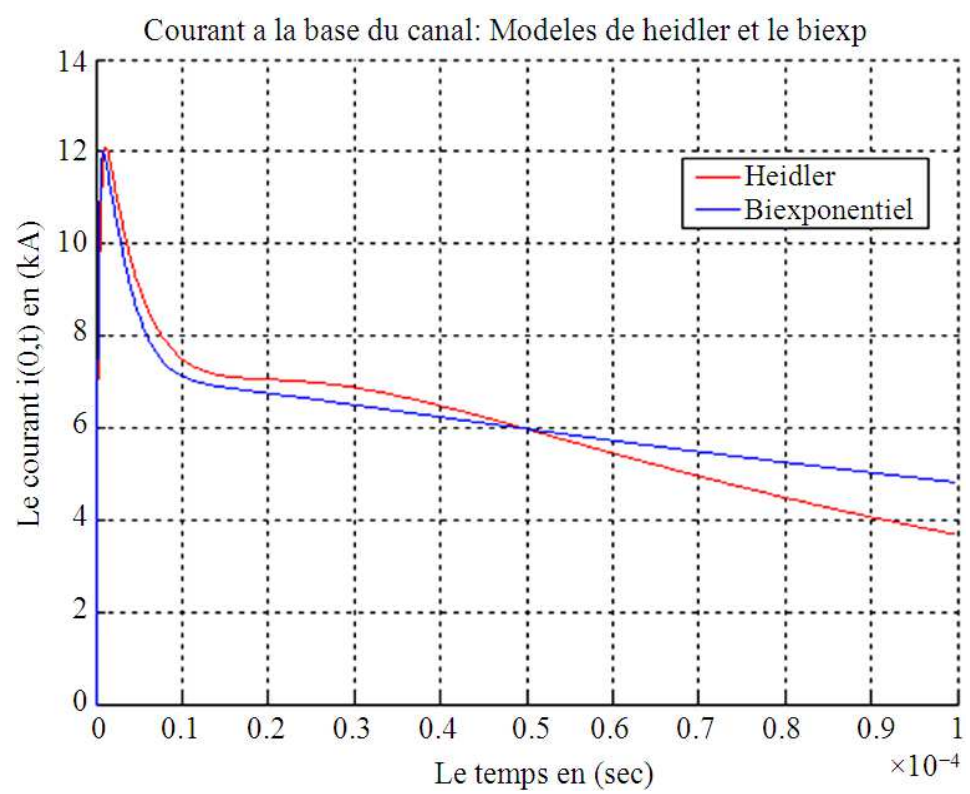

Fig. 2. Current at the base of Lightning channel with Heidler and double exponential models (SIMLIGHTNING 2012)

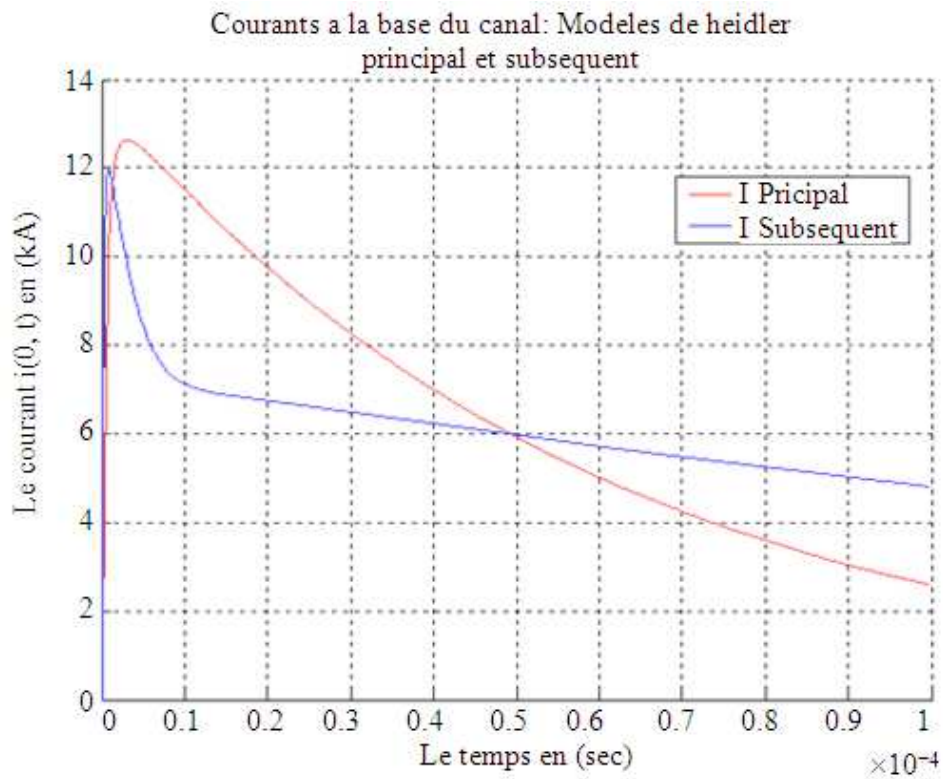

Fig. 3. Principal and subsequent strokes of the Current at the base of Lightning channel with Heidler model (SIMLIGHTNING 2012)

Table 1. Parameters for the typical current waveform at the base of a lightning used in Heidler model

\begin{tabular}{|c|c|c|c|c|c|c|c|c|c|c|}
\hline & I1 kA & $\mathrm{t} 11 \mathrm{~ms}$ & $\mathrm{t} 12 \mathrm{~ms}$ & $\mathrm{n} 1$ & I2 kA & $\mathrm{t} 21 \mathrm{~ms}$ & $\mathrm{t} 22 \mathrm{~ms}$ & $\mathrm{n} 2$ & $1 \mathrm{~km}$ & $\mathrm{v} \mathrm{m} / \mathrm{s}$ \\
\hline Case1 & 17.0 & 0.4 & 4.0 & 2 & 8 & 4 & 50 & 2 & 1.5 & $1.0 \times 10^{8}$ \\
\hline Case2 & 10.5 & 2.0 & 4.8 & 2 & 9 & 20 & 26 & 2 & 2.0 & $1.5 \times 10^{8}$ \\
\hline Case3 & 19.5 & 1.0 & 2.0 & 2 & 12 & 8 & 30 & 2 & 1.5 & $1.0 \times 10^{8}$ \\
\hline
\end{tabular}




$$
\begin{aligned}
& \int_{0}^{\mathrm{t}} \mathrm{i}\left(\mathrm{z}^{\prime}, \mathrm{t}-\frac{\mathrm{R}}{\mathrm{C}}\right) \mathrm{d} \tau=\frac{\mathrm{I}_{\mathrm{o}}}{\alpha}\left(1-\mathrm{e}^{\alpha \mathrm{t}}\right) \exp \left[\frac{\alpha \mathrm{R}}{\mathrm{c}}+\left(\frac{\alpha}{\mathrm{v}}-\frac{1}{\lambda}\right) \mathrm{z}^{\prime}\right] \\
& -\frac{\mathrm{I}_{\mathrm{o}}}{\beta}\left(1-\mathrm{e}^{\beta \mathrm{t}}\right) \exp \left[\frac{\beta \mathrm{R}}{\mathrm{c}}+\left(\frac{\beta}{\mathrm{v}}-\frac{1}{\lambda}\right) \mathrm{z}^{\prime}\right] \\
& \frac{\partial \mathrm{i}}{\partial \mathrm{t}}=\mathrm{I}_{\mathrm{o}}\left\{\begin{array}{l}
-\alpha \mathrm{e}^{-\alpha \mathrm{t}} \exp \left[\frac{\alpha \mathrm{R}}{\mathrm{c}}+\left(\frac{\alpha}{\mathrm{v}}-\frac{1}{\lambda}\right) \mathrm{z}^{\prime}\right] \\
+\beta \mathrm{e}^{-\beta \mathrm{t}} \exp \left[\frac{\beta \mathrm{R}}{\mathrm{c}}+\left(\frac{\beta}{\mathrm{v}}-\frac{1}{\lambda}\right) \mathrm{z}^{\prime}\right]
\end{array}\right\}
\end{aligned}
$$

Numerical approaches of trapez integration Equation 10 and 11:

$$
\begin{aligned}
& \int_{0}^{t} \mathrm{i}\left(\mathrm{z}^{\prime}, \tau-\frac{\mathrm{R}}{\mathrm{c}}\right) \mathrm{d} \tau \cong \mathrm{k}\left[\frac{1}{2} \mathrm{i}\left(\mathrm{z}^{\prime}, \tau-\frac{\mathrm{R}}{\mathrm{c}}\right)+\right. \\
& \left.\sum_{\mathrm{j}=1}^{\mathrm{j}=\mathrm{n}-1} \mathrm{i}\left(\mathrm{z}^{\prime}, j \mathrm{j}-\frac{\mathrm{R}}{\mathrm{c}}\right)+\frac{1}{2} \mathrm{i}\left(\mathrm{z}^{\prime}, \mathrm{t}-\frac{\mathrm{R}}{\mathrm{c}}\right)\right] \\
& \int_{0}^{\mathrm{t}} \mathrm{i}\left(-\mathrm{z}^{\prime}, \tau-\frac{\tilde{R}}{\mathrm{c}}\right) \mathrm{d} \tau \cong \mathrm{k}\left[\frac{1}{2} \mathrm{i}\left(\mathrm{z}^{\prime},-\frac{\tilde{\mathrm{R}}}{\mathrm{c}}\right)\right. \\
& \left.+\sum_{j=1}^{\mathrm{j}=\mathrm{n}-1} \mathrm{i}\left(\mathrm{z}^{\prime}, \mathrm{jk}-\frac{\tilde{\mathrm{R}}}{\mathrm{c}}\right)+\frac{1}{2} \mathrm{i}\left(-z^{\prime}, \tau-\frac{\tilde{R}}{\mathrm{c}}\right)\right]
\end{aligned}
$$

Numerical Method of the operator of first order differences Equation (12 and 13):

$$
\frac{\partial \mathrm{i}\left(\mathrm{z}^{\prime}, \mathrm{t}-\frac{\mathrm{R}}{\mathrm{c}}\right)}{\partial \mathrm{t}} \mathrm{z}_{\mathrm{m}} \simeq \frac{\mathrm{i}\left(\mathrm{z}_{\mathrm{m}}, \mathrm{t}-\frac{\mathrm{R}}{\mathrm{c}}\right)-\mathrm{i}\left(\mathrm{z}_{\mathrm{m}}, \mathrm{t}-\mathrm{k}-\frac{\mathrm{R}}{\mathrm{c}}\right)}{\mathrm{k}}
$$

\subsection{Horizontal Electrical Field}

$$
\mathrm{E}_{\mathrm{r}}(\mathrm{r}, \mathrm{z}, \mathrm{t})=\frac{1}{4 \pi \varepsilon_{0}}\left[\begin{array}{l}
{\left[\begin{array}{l}
\int_{0}^{\mathrm{H}} \frac{3 \mathrm{r}\left(\mathrm{z}-\mathrm{z}^{\prime}\right)}{\mathrm{R}^{5}} \int_{0}^{\mathrm{t}} \mathrm{i}\left(\mathrm{z}^{\prime} \tau-\frac{\mathrm{R}}{\mathrm{c}}\right) \mathrm{d} \tau \mathrm{d} \mathrm{z}^{\prime} \\
\left.+\int_{0}^{\mathrm{H}} \frac{3 \mathrm{r}\left(\mathrm{z}+\mathrm{z}^{\prime}\right)}{\tilde{\mathrm{R}}^{5}} \int_{0}^{\mathrm{t}} \mathrm{i}\left(-\mathrm{z}^{\prime}, \tau-\frac{\tilde{\mathrm{R}}}{\mathrm{c}}\right) \mathrm{d} \tau \mathrm{d} \mathrm{z}^{\prime}\right]
\end{array}\right]} \\
{\left[\begin{array}{l}
\left.\int_{0}^{\mathrm{H}} \frac{3 \mathrm{r}\left(\mathrm{z}+\mathrm{z}^{\prime}\right)}{\mathrm{cR}} \mathrm{i}\left(\mathrm{z}^{\prime}, \mathrm{t}-\frac{\mathrm{R}}{\mathrm{c}}\right) \mathrm{dz} \mathrm{z}^{\prime}\right] \\
+\int_{0}^{\mathrm{H}} \frac{3 \mathrm{r}\left(\mathrm{z}+\mathrm{z}^{\prime}\right)}{\mathrm{c} \tilde{\mathrm{R}}^{4}} \mathrm{i}\left(-\mathrm{z}^{\prime}, \mathrm{t}-\frac{\tilde{\mathrm{R}}}{\mathrm{c}}\right) \mathrm{dz} \mathrm{z}^{\prime}
\end{array}\right]} \\
{\left[\begin{array}{l}
\int_{0}^{\mathrm{H}} \frac{\mathrm{r}^{2}}{\mathrm{c}^{2} \mathrm{R}^{3}} \frac{\partial \mathrm{i}\left(\mathrm{z}^{\prime}, \mathrm{t}-\frac{\mathrm{R}}{\mathrm{c}}\right)}{\partial \mathrm{t}} \mathrm{dz} \mathrm{z}^{\prime} \\
+\int_{0}^{\mathrm{H}} \frac{\mathrm{r}^{2}}{\mathrm{c}^{2} \tilde{\mathrm{R}}^{3}} \frac{\partial \mathrm{i}\left(\mathrm{z}^{\prime}, \mathrm{t}-\frac{\tilde{\mathrm{R}}}{\mathrm{c}}\right)}{\partial \mathrm{t}} \mathrm{dz} \mathrm{z}^{\prime}
\end{array}\right]}
\end{array}\right]
$$

$$
\mathrm{E}_{\mathrm{r}}(\mathrm{r}, \mathrm{z}, \mathrm{t})=\left[\mathrm{S}_{1}+\mathrm{S}_{2}+\mathrm{S}_{3}\right]
$$

$$
\mathrm{S}_{1}=\frac{1}{4 \pi \varepsilon_{0}}\left[\frac{3 \mathrm{rk}}{2}\left[\mathrm{I}_{1}+\mathrm{I}_{2}+\tilde{\mathrm{I}}_{1}+\tilde{\mathrm{I}}_{2}\right]+3 \mathrm{rk} \sum_{\mathrm{j}=1}^{\mathrm{j}=\mathrm{n}-1}\left(\mathrm{~L}_{\mathrm{j}}+\tilde{\mathrm{L}}_{\mathrm{j}}\right)\right]
$$

And:

$$
\begin{aligned}
& \mathrm{I}_{1}=\int_{0}^{\mathrm{H}} \frac{\left(\mathrm{z}-\mathrm{z}^{\prime}\right)}{\mathrm{R}^{5}} \mathrm{i}\left(\mathrm{z}^{\prime},-\frac{\mathrm{R}}{\mathrm{c}}\right) \mathrm{d} \mathrm{z}^{\prime} \cong \mathrm{h}\left[\frac{1}{2} \frac{\mathrm{z}}{\mathrm{R}_{0}^{5}} \mathrm{i}\left(0,-\frac{\mathrm{R}_{\mathrm{o}}}{\mathrm{c}}\right)\right. \\
& \left.+\sum_{\mathrm{m}=1}^{\mathrm{m}=\mathrm{N}-1} \frac{(\mathrm{z}-\mathrm{mh})}{\mathrm{R}_{\mathrm{m}}^{5}} \mathrm{i}\left(\mathrm{mh},-\frac{\mathrm{R}_{\mathrm{m}}}{\mathrm{c}}\right)+\frac{1}{2} \frac{(\mathrm{z}-\mathrm{H})}{\mathrm{R}_{\mathrm{N}}^{5}} \mathrm{i}\left(\mathrm{H},-\frac{\mathrm{R}_{\mathrm{N}}}{\mathrm{c}}\right)\right]
\end{aligned}
$$$$
I_{2}=\int_{0}^{H} \frac{\left(z-z^{\prime}\right)}{R^{5}} i\left(z^{\prime}, t-\frac{R}{c}\right) d z^{\prime} h\left[\frac{1}{2} \frac{z}{R_{0}^{5}} i\left(0, t-\frac{R_{0}}{c}\right)\right.
$$$$
\left.+\sum_{\mathrm{m}=1}^{\mathrm{m}=\mathrm{N}-1} \frac{(\mathrm{z}-\mathrm{mh})}{\mathrm{R}_{\mathrm{m}}^{5}} \mathrm{i}\left(\mathrm{mh}, \mathrm{t}-\frac{\mathrm{R}_{\mathrm{m}}}{\mathrm{c}}\right)+\frac{1}{2} \frac{(\mathrm{z}-\mathrm{H})}{\mathrm{R}_{\mathrm{N}}^{5}} \mathrm{i}\left(\mathrm{H}, \mathrm{t}-\frac{\mathrm{R}_{\mathrm{N}}}{\mathrm{c}}\right)\right]
$$$$
\tilde{\mathrm{I}}_{1}=\int_{0}^{\mathrm{H}} \frac{\left(\mathrm{z}+\mathrm{z}^{\prime}\right)}{\tilde{\mathrm{R}}^{5}} \mathrm{i}\left(-\mathrm{z}^{\prime},-\frac{\tilde{\mathrm{R}}}{\mathrm{c}}\right) \mathrm{dz^{ \prime }} \cong \mathrm{h}\left[\frac{1}{2} \frac{\mathrm{z}}{\tilde{\mathrm{R}}_{0}^{5}} \mathrm{i}\left(0,-\frac{\tilde{\mathrm{R}}_{0}}{\mathrm{c}}\right)\right.
$$$$
\left.+\sum_{\mathrm{m}=1}^{\mathrm{m}=\mathrm{N}-1} \frac{(\mathrm{z}+\mathrm{mh})}{\tilde{\mathrm{R}}_{\mathrm{m}}^{5}} \mathrm{i}\left(-\mathrm{mh},-\frac{\tilde{\mathrm{R}}_{\mathrm{m}}}{\mathrm{c}}\right)+\frac{1}{2} \frac{(\mathrm{z}+\mathrm{H})}{\tilde{\mathrm{R}}_{\mathrm{m}}^{5}} \mathrm{i}\left(-\mathrm{H},-\frac{\tilde{\mathrm{R}}_{\mathrm{N}}}{\mathrm{c}}\right)\right]
$$

$$
\begin{aligned}
& \tilde{\mathrm{I}}_{2}=\int_{0}^{\mathrm{H}} \frac{\left(\mathrm{z}+\mathrm{z}^{\prime}\right)}{\tilde{\mathrm{R}}^{5}} \mathrm{i}\left(-\mathrm{z}^{\prime}, \mathrm{t}-\frac{\tilde{\mathrm{R}}}{\mathrm{c}}\right) \mathrm{dz} \mathrm{z}^{\prime} \cong \mathrm{h}\left[\frac{1}{2} \frac{\mathrm{z}}{\tilde{\mathrm{R}}_{0}^{5}} \mathrm{i}\left(0,-\frac{\tilde{\mathrm{R}}_{0}}{\mathrm{c}}\right)\right. \\
& \left.+\sum_{\mathrm{m}=1}^{\mathrm{m}=\mathrm{N}-1} \frac{(\mathrm{z}+\mathrm{mh})}{\tilde{\mathrm{R}}_{\mathrm{m}}^{5}} \mathrm{i}\left(-\mathrm{mh}, \mathrm{t}-\frac{\tilde{\mathrm{R}}_{\mathrm{m}}}{\mathrm{c}}\right)+\frac{1}{2} \frac{(\mathrm{z}+\mathrm{H})}{\tilde{\mathrm{R}}_{\mathrm{N}}^{5}} \mathrm{i}\left(-\mathrm{H},-\frac{\tilde{\mathrm{R}}_{\mathrm{N}}}{\mathrm{c}}\right)\right]
\end{aligned}
$$

$$
\begin{aligned}
& \mathrm{L}_{\mathrm{j}}=\int_{0}^{\mathrm{H}} \frac{\left(\mathrm{z}-\mathrm{z}^{\prime}\right)}{\mathrm{R}^{5}} \mathrm{i}\left(\mathrm{z}^{\prime}, \mathrm{jk}-\frac{\mathrm{R}}{\mathrm{c}}\right) \mathrm{dz} \mathrm{z}^{\prime} \cong \mathrm{h}\left[\frac{1}{2} \frac{\mathrm{z}}{\mathrm{R}_{0}^{5}} \mathrm{i}\left(0, j \mathrm{k}-\frac{\mathrm{R}_{0}}{\mathrm{c}}\right)\right. \\
& \left.+\sum_{\mathrm{m}=1}^{\mathrm{m}=\mathrm{N}-1} \frac{(\mathrm{z}-\mathrm{mh})}{\mathrm{R}_{\mathrm{m}}^{5}} \mathrm{i}\left(-\mathrm{mh}, \mathrm{t}-\frac{\mathrm{R}_{\mathrm{m}}}{\mathrm{c}}\right)+\frac{1}{2} \frac{(\mathrm{z}+\mathrm{H})}{\mathrm{R}_{\mathrm{N}}^{5}} \mathrm{i}\left(-\mathrm{H}, \mathrm{t}-\frac{\mathrm{R}_{\mathrm{N}}}{\mathrm{c}}\right)\right]
\end{aligned}
$$

$$
\tilde{\mathrm{L}}_{\mathrm{j}}=\int_{0}^{\mathrm{H}} \frac{\left(\mathrm{z}+\mathrm{z}^{\prime}\right)}{\tilde{\mathrm{R}}^{5}} \mathrm{i}\left(-\mathrm{z}^{\prime}, \mathrm{jk}-\frac{\tilde{\mathrm{R}}}{\mathrm{c}}\right) \mathrm{dz} \mathrm{z}^{\prime} \cong \mathrm{h}\left[\frac{1}{2} \frac{\mathrm{z}}{\tilde{\mathrm{R}}_{0}^{5}} \mathrm{i}\left(0, j \mathrm{k}-\frac{\tilde{\mathrm{R}}_{0}}{\mathrm{c}}\right)\right.
$$$$
\left.+\sum_{\mathrm{m}=1}^{\mathrm{m}=\mathrm{N}-1} \frac{(\mathrm{z}+\mathrm{mh})}{\tilde{\mathrm{R}}_{\mathrm{m}}^{5}} \mathrm{i}\left(-\mathrm{mh}, \mathrm{jk}-\frac{\tilde{\mathrm{R}}_{\mathrm{m}}}{\mathrm{c}}\right)+\frac{1}{2} \frac{(\mathrm{z}+\mathrm{H})}{\mathrm{R}_{\mathrm{N}}^{5}} \mathrm{i}\left(-\mathrm{H}, \mathrm{jk}-\frac{\tilde{\mathrm{R}}_{\mathrm{N}}}{\mathrm{c}}\right)\right]
$$

$$
\mathrm{S}_{1}=\frac{3 \mathrm{rk}}{4 \pi \varepsilon_{0}}\left[\frac{\mathrm{I}_{1}+\mathrm{I}_{2}+\tilde{\mathrm{I}}_{1}+\tilde{\mathrm{I}}_{2}}{2}+\sum_{\mathrm{j}=1}^{\mathrm{j}=\mathrm{n}-1}\left(\mathrm{~L}_{\mathrm{j}}+\tilde{\mathrm{L}}_{\mathrm{j}}\right)\right]
$$
(14):

Similarly find the expressions of S2 and S3 Equation 


$$
\begin{aligned}
& \mathrm{S}_{2} \simeq \frac{3 \mathrm{rh}}{4 \mathrm{c} \pi \varepsilon_{0}}\left[\begin{array}{l}
{\left[\frac{1}{2} \frac{\mathrm{z}}{\mathrm{R}_{0}^{4}} \mathrm{i}\left(0, \mathrm{t}-\frac{\mathrm{R}_{0}}{\mathrm{c}}\right)+\sum_{\mathrm{m}=1}^{\mathrm{m}=\mathrm{N}-1} \frac{(\mathrm{z}-\mathrm{mh})}{\mathrm{R}_{\mathrm{m}}^{4}} \mathrm{i}\left(-\mathrm{mh}, \mathrm{t}-\frac{\mathrm{R}_{\mathrm{m}}}{\mathrm{c}}\right)+\frac{1}{2} \frac{(\mathrm{z}-\mathrm{H})}{\mathrm{R}_{\mathrm{N}}^{4}} \mathrm{i}\left(\mathrm{H}, \mathrm{t}-\frac{\mathrm{R}_{\mathrm{N}}}{\mathrm{c}}\right)\right]} \\
\left.+\left[\frac{1}{2} \frac{\mathrm{z}}{\tilde{\mathrm{R}}_{0}^{4}} \mathrm{i}\left(0, \mathrm{t}-\frac{\tilde{\mathrm{R}}_{0}}{\mathrm{c}}\right)+\sum_{\mathrm{m}=1}^{\mathrm{m}=\mathrm{N}-1} \frac{(\mathrm{z}+\mathrm{mh})}{\tilde{\mathrm{R}}_{\mathrm{m}}^{4}} \mathrm{i}\left(-\mathrm{mh}, \mathrm{t}-\frac{\tilde{\mathrm{R}} \mathrm{m}}{\mathrm{c}}\right)+\frac{1}{2} \frac{(\mathrm{z}+\mathrm{H})}{\mathrm{R}_{\mathrm{N}}^{4}} \mathrm{i}\left(\mathrm{H}, \mathrm{t}-\frac{\tilde{\mathrm{R}}_{\mathrm{N}}}{\mathrm{c}}\right)\right]\right]
\end{array}\right]
\end{aligned}
$$

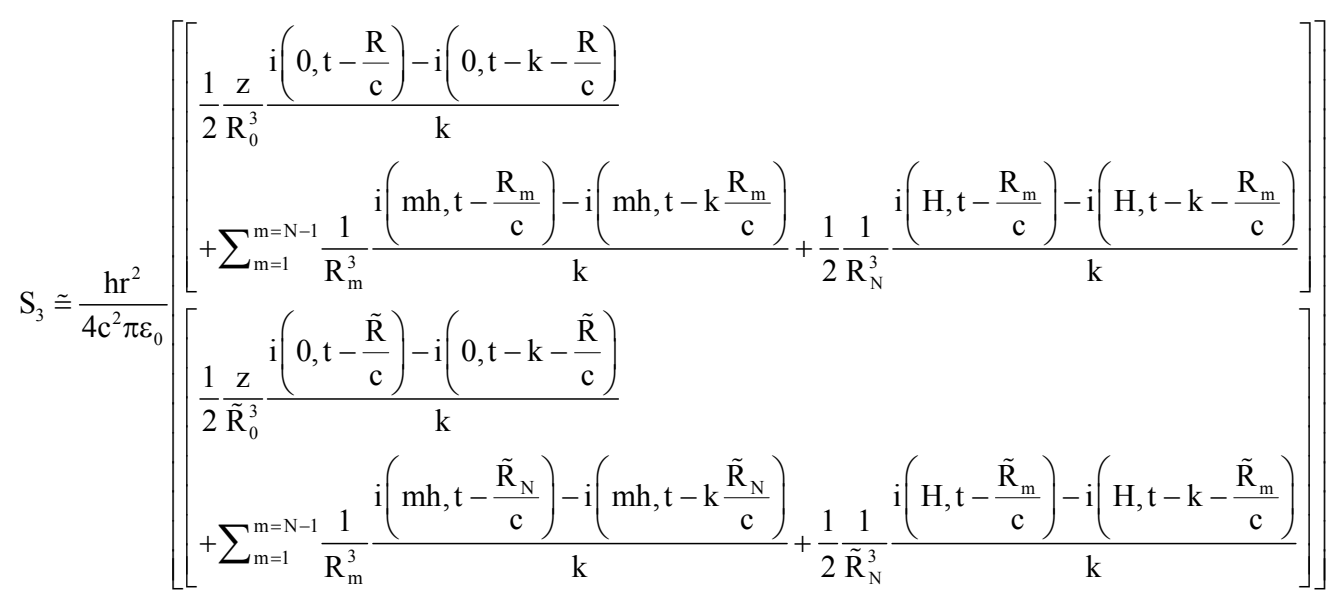

$\operatorname{Er}(\mathrm{r}, \mathrm{z}, \mathrm{t}) \cong \mathrm{S}_{1}+\mathrm{S}_{2}+\mathrm{S}_{3}$

\subsection{Vertical Electrical Field}

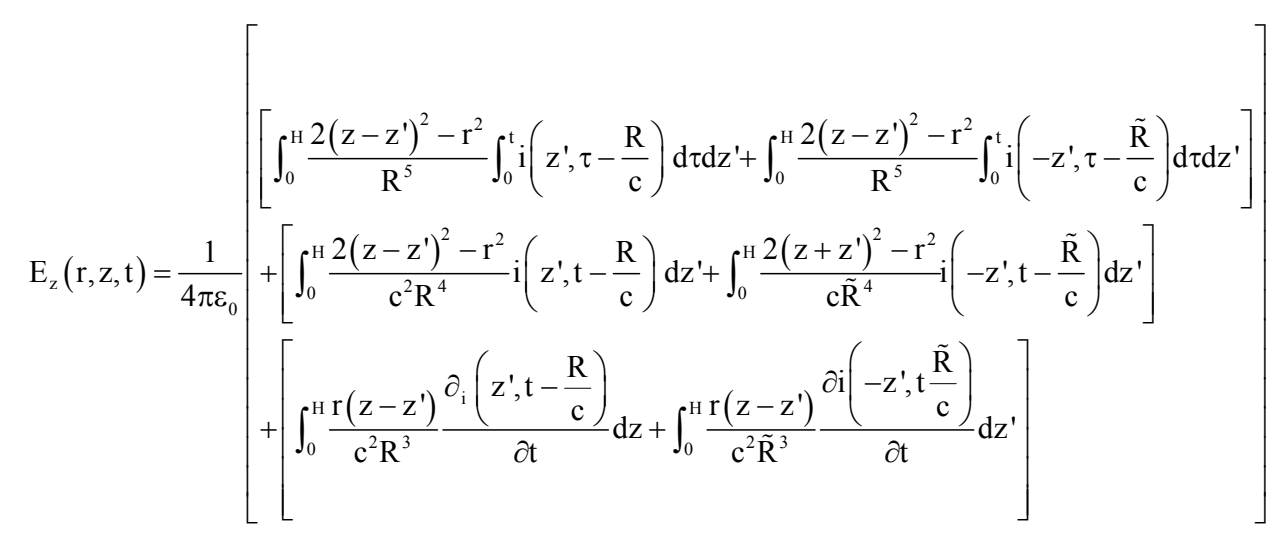

$E_{z}(r, z, t)$ is the sum of three terms or field components Equation (15):

$$
\begin{gathered}
\mathrm{E}_{\mathrm{Z}}(\mathrm{r}, \mathrm{Z}, \mathrm{t})=\cong \mathrm{S}_{1}^{\prime}+\mathrm{S}_{2}^{\prime}+\mathrm{S}_{3}^{\prime} \\
\mathrm{S}_{1}^{\prime}=\frac{1}{4 \pi \varepsilon_{0}}\left[\frac{\mathrm{J}_{1}+\mathrm{J}_{2}+\tilde{\mathrm{J}}_{1}+\mathrm{j}_{2}}{2}+\sum_{\mathrm{j}=1}^{\mathrm{j}=\mathrm{n}-1}\left(\mathrm{M}_{\mathrm{j}}+\tilde{\mathrm{M}}_{\mathrm{j}}\right)\right]
\end{gathered}
$$




$$
\begin{aligned}
& \mathrm{S}_{2}^{\prime} \cong \frac{\mathrm{h}}{4 \mathrm{c} \pi \varepsilon_{0}}\left[\begin{array}{l}
{\left[\frac{1}{2} \frac{2 \mathrm{z}^{2}-\mathrm{r}^{2}}{\mathrm{R}_{0}^{4}} \mathrm{i}\left(0, \mathrm{t}-\frac{\mathrm{R}_{0}}{\mathrm{c}}\right)+\sum_{\mathrm{m}=1}^{\mathrm{m}=\mathrm{N}-1} \frac{2(\mathrm{z}-\mathrm{mh})^{2}-\mathrm{r}^{2}}{\mathrm{R}_{\mathrm{m}}^{4}} \mathrm{i}\left(\mathrm{mh}, \mathrm{t}-\frac{\mathrm{R}_{\mathrm{m}}}{\mathrm{c}}\right)+\frac{1}{2} \frac{(\mathrm{z}-\mathrm{H})^{2}}{\mathrm{R}_{\mathrm{N}}^{4}} \mathrm{i}\left(\mathrm{H}, \mathrm{t}-\frac{\mathrm{R}_{\mathrm{N}}}{\mathrm{c}}\right)\right]} \\
\left.+\left[\frac{1}{2} \frac{2 \mathrm{z}^{2}-\mathrm{r}^{2}}{\tilde{\mathrm{R}}_{0}^{4}} \mathrm{i}\left(0, \mathrm{t}-\frac{\tilde{\mathrm{R}}_{0}}{\mathrm{c}}\right)+\sum_{\mathrm{m}=1}^{\mathrm{m}=\mathrm{N}-1} \frac{2(\mathrm{z}-\mathrm{mh})^{2}-\mathrm{r}^{2}}{\tilde{\mathrm{R}}_{\mathrm{m}}^{4}} \mathrm{i}\left(\mathrm{mh}, \mathrm{t}-\frac{\tilde{\mathrm{R}}_{\mathrm{m}}}{\mathrm{c}}\right)+\frac{1}{2} \frac{2(\mathrm{z}-\mathrm{mh})^{2}-\mathrm{r}^{2}}{\tilde{\mathrm{R}}_{\mathrm{N}}^{4}} \mathrm{i}\left(-\mathrm{H}, \mathrm{t}-\frac{\tilde{\mathrm{R}}_{\mathrm{N}}}{\mathrm{c}}\right)\right]\right]
\end{array}\right]
\end{aligned}
$$

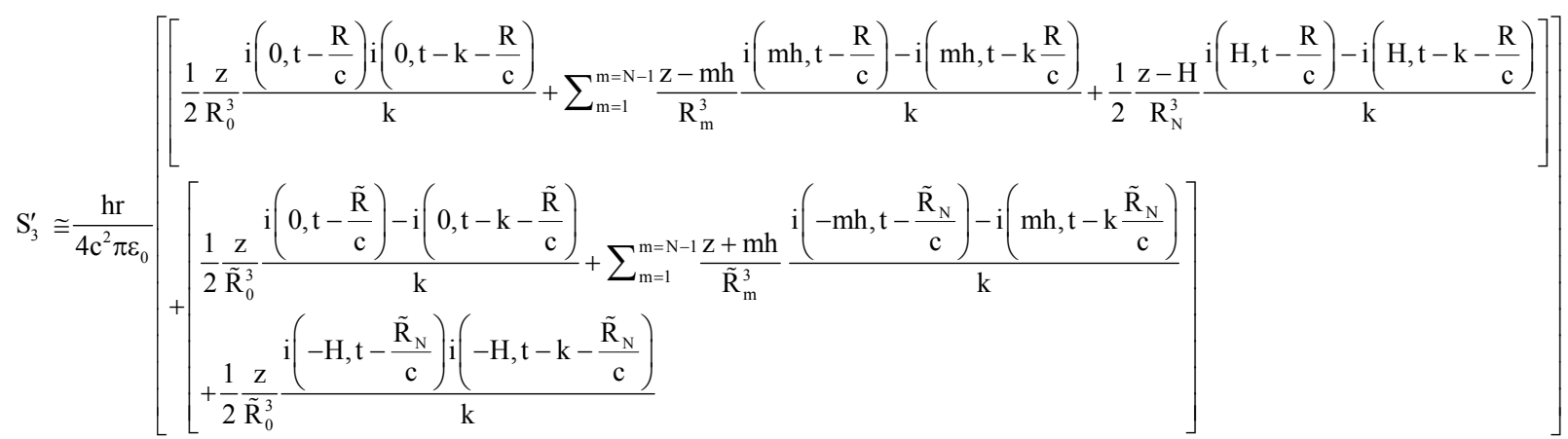

$\mathrm{E}_{\mathrm{z}}(\mathrm{r}, \mathrm{z}, \mathrm{t})=\cong \mathrm{S}_{1}^{\prime}+\mathrm{S}_{2}^{\prime}+\mathrm{S}_{3}^{\prime}$

S1 and $\mathrm{S}_{1}{ }_{1}=$ The electrostatic component in the Electrical field

$\mathrm{S} 2$ and $\mathrm{S}_{2}{ }_{2}=$ The electric radiation component in the Electrical field

$\mathrm{S} 3$ and $\mathrm{S}_{3}{ }_{3}=$ The magnetic induction component in the Electrical field

\subsection{Azimutal Magnetic Field Equation (16)}

$$
\begin{aligned}
& \mathrm{B} \phi(\mathrm{r}, \mathrm{z}, \mathrm{t})=\frac{\mu_{0}}{4 \pi}\left[\left[\int_{0}^{\mathrm{H}} \frac{\mathrm{r}}{\mathrm{R}^{3}} \mathrm{i}\left(\mathrm{z}^{\prime}, \tau-\frac{\mathrm{R}}{\mathrm{c}}\right) \mathrm{dz} \mathrm{z}^{\prime}+\int_{0}^{\mathrm{H}} \frac{\mathrm{r}}{\tilde{\mathrm{R}}^{3}} \mathrm{i}\left(-\mathrm{z}^{\prime} \tau-\frac{\tilde{\mathrm{R}}}{\mathrm{c}}\right) \mathrm{dz} \mathrm{z}^{\prime}\right]+\left[\int_{0}^{\mathrm{H}} \frac{\mathrm{r}}{\mathrm{cR} \mathrm{R}^{2}} \frac{\partial \mathrm{i}\left(\mathrm{z}^{\prime}, \mathrm{t}-\frac{\mathrm{R}}{\mathrm{c}}\right)}{\partial \mathrm{t}} \mathrm{dz}+\int_{0}^{\mathrm{H}} \frac{\mathrm{r}}{\mathrm{c} \tilde{\mathrm{R}}^{2}} \frac{\partial \mathrm{i}\left(-\mathrm{z}^{\prime}, \mathrm{t}-\frac{\tilde{\mathrm{R}}}{\mathrm{c}}\right)}{\partial \mathrm{t}} \mathrm{dz} \mathrm{z}^{\prime}\right]\right] \\
& \mathrm{S}_{1}^{\prime \prime} \cong \frac{\mathrm{h} \mu_{0} \mathrm{r}}{4 \pi}\left[\begin{array}{l}
{\left[\frac{1}{2} \frac{1}{\mathrm{R}_{0}^{3}} \mathrm{i}\left(0, \mathrm{t}-\frac{\mathrm{R}_{0}}{\mathrm{c}}\right)+\sum_{\mathrm{m}=1}^{\mathrm{m}=\mathrm{N}-1} \frac{1}{\mathrm{R}_{\mathrm{m}}^{3}} \mathrm{i}\left(\mathrm{mh}, \mathrm{t}-\frac{\mathrm{R}_{\mathrm{m}}}{\mathrm{c}}\right)+\frac{1}{2} \frac{1}{\mathrm{R}_{\mathrm{N}}^{3}} \mathrm{i}\left(\mathrm{H}, \mathrm{t}-\frac{\mathrm{R}_{\mathrm{N}}}{\mathrm{c}}\right)\right]} \\
\left.+\left[\frac{1}{2} \frac{1}{\mathrm{R}_{0}^{3}} \mathrm{i}\left(0, \mathrm{t}-\frac{\tilde{\mathrm{R}}_{0}}{\mathrm{c}}\right)+\sum_{\mathrm{m}=1}^{\mathrm{m}=\mathrm{N}-1} \frac{1}{\tilde{\mathrm{R}}_{\mathrm{m}}^{3}} \mathrm{i}\left(-\mathrm{mh}, \mathrm{t}-\frac{\tilde{\mathrm{R}}_{\mathrm{m}}}{\mathrm{c}}\right)+\frac{1}{2} \frac{1}{\tilde{\mathrm{R}}_{\mathrm{N}}^{3}} \mathrm{i}\left(\mathrm{H}, \mathrm{t}-\frac{\tilde{\mathrm{R}}_{\mathrm{N}}}{\mathrm{c}}\right)\right]\right]
\end{array}\right]
\end{aligned}
$$

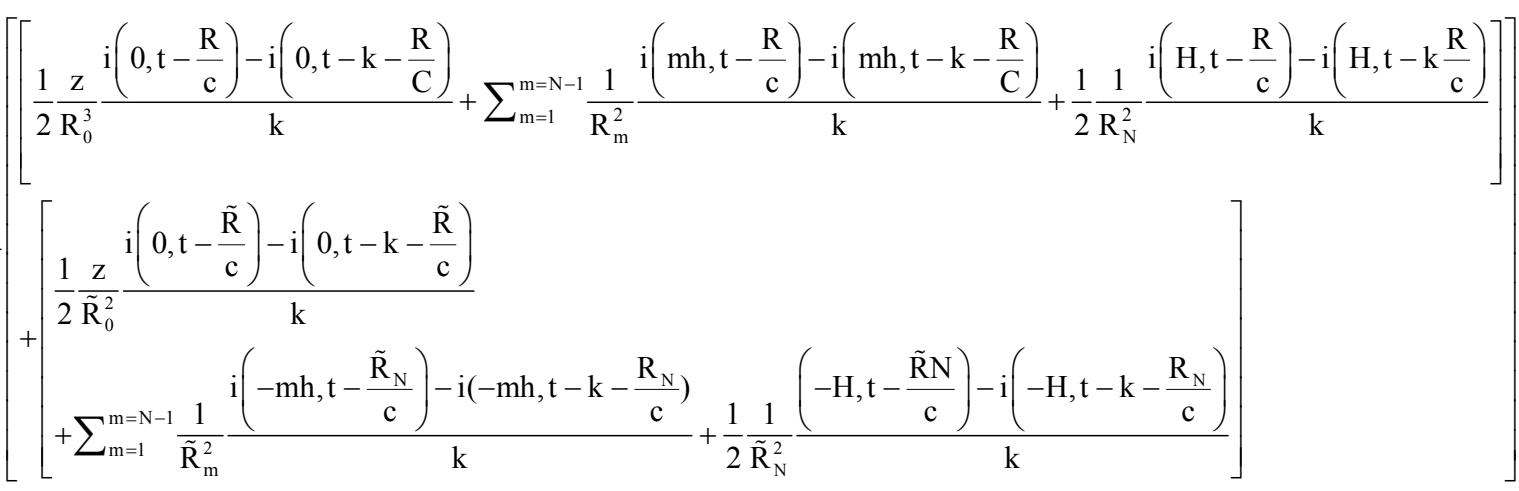


$\mathrm{B}_{\phi}(\mathrm{r}, \mathrm{z}, \mathrm{t})=\mathrm{S}_{1}+\mathrm{S}_{2}$

$\mathrm{S}{ }_{1}=$ The electric radiation component in the magnetic field

$\mathrm{S}{ }_{2}=$ The magnetic induction component in the magnetic field

\section{SIMLIGHTNING CODE}

After having developed a program with over 350 lines in Fortran, it has been implemented as a module (code) into Matlab in Version 2011 considering all the parameters electrical, magnetic and geometric expressions involved in the proposed new model of electromagnetic fields and all approximations required by the mathematical tools used. This program named SIMLIGHTNING code and his principal function is to simulate the current in lightning channel and the lightning electromagnetic fields with the following variables:

- The current at the base of the channel by lightning two types of functions

- The return current in the channel model by the lightning MTLE

- The 08 components of the three fields radiated by lightning
- The 03 vectors of electromagnetic fields radiated by the lightning Canal: Electric vertical, horizontal electric and azimuth magnetic

\subsection{Results of SIMLIGHTNING Code Show in Fig. 2, 3, 4, 6, 7, 9, 10 and 11}

\subsubsection{Analysis Results}

The results obtained by simulation are compared with those obtained by experimental measurements and other results published by other authors cited in references:

- For electric currents generated by lightning, the results illustrated in (Fig. 2, 3, 6 and 7) by SIMLIGHTNING have high concordance with those figures obtained by measurements (Fig. 5) in (Heidler and Hopf, 1994; Nucci and Rachidi, 1989; Rachidi et al., 1992) or published (Fig. 8) in (Cooray, 1994; 1998)

- For the results of the electromagnetic field obtained by SIMLIGHTNING for its three components on the basis of the proposed new model in (Fig. 9-14) the agreement was satisfactory compared to measurements and results published in (Cooray, 1994; 1998; 2001) particular for the electric field where agreement was better than that of the magnetic field

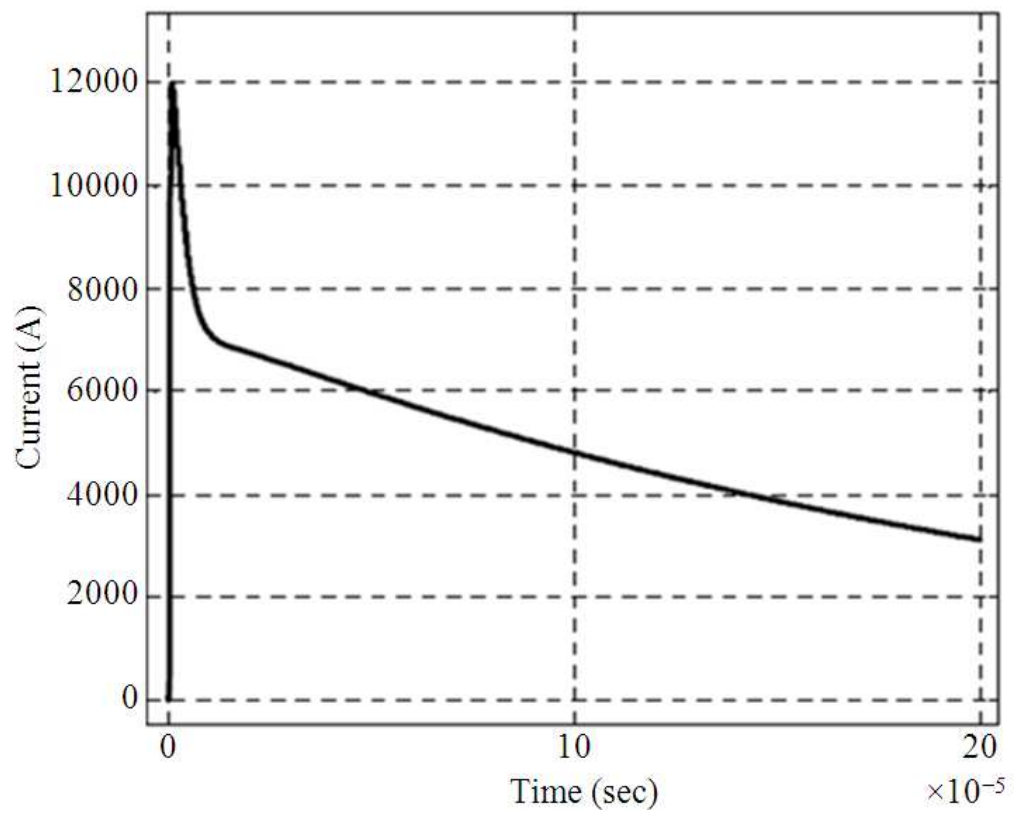

Fig. 4. Current at the base of Lightning channel with Heidler model (Verbanov, 2004) 
Dib Djalel et al. / American Journal of Engineering and Applied Science 6 (4): 369-383, 2013

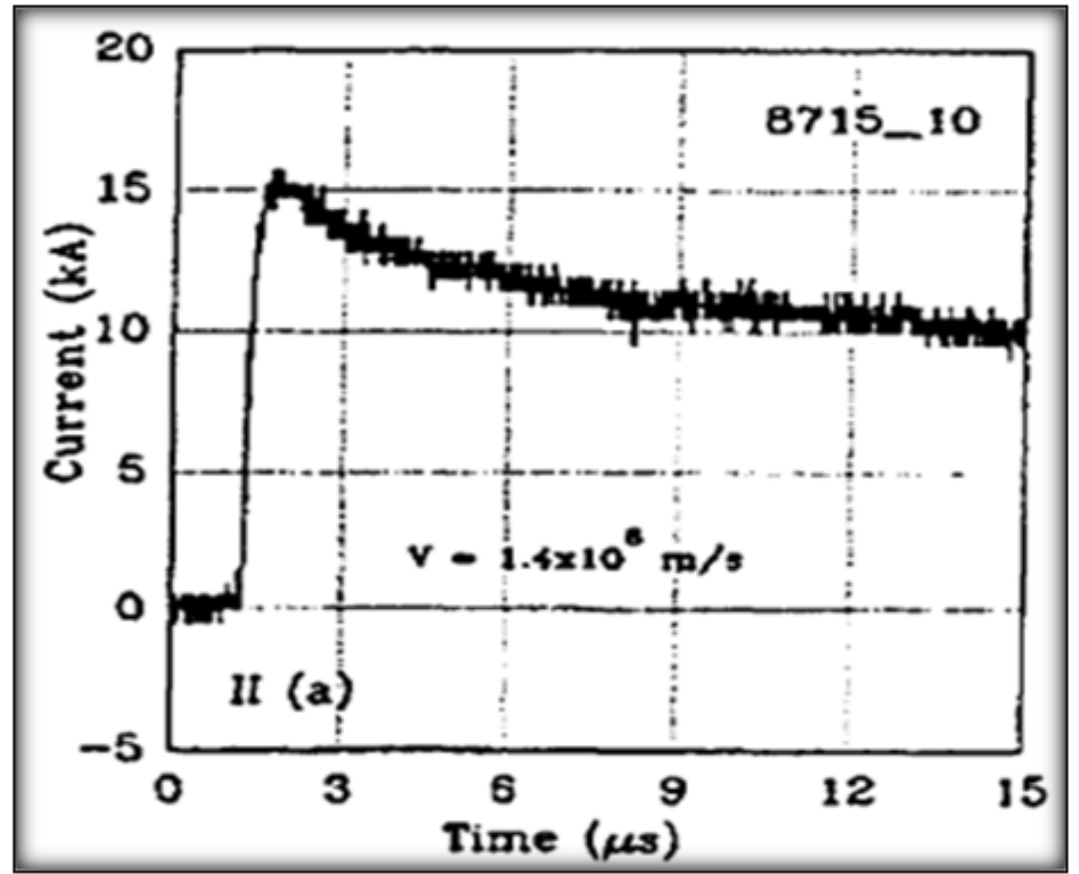

Fig. 5. Current at the base of Lightning channel Experiment measurement (Nucci and Rachidi, 1989; Rachidi et al., 1992; 1998)

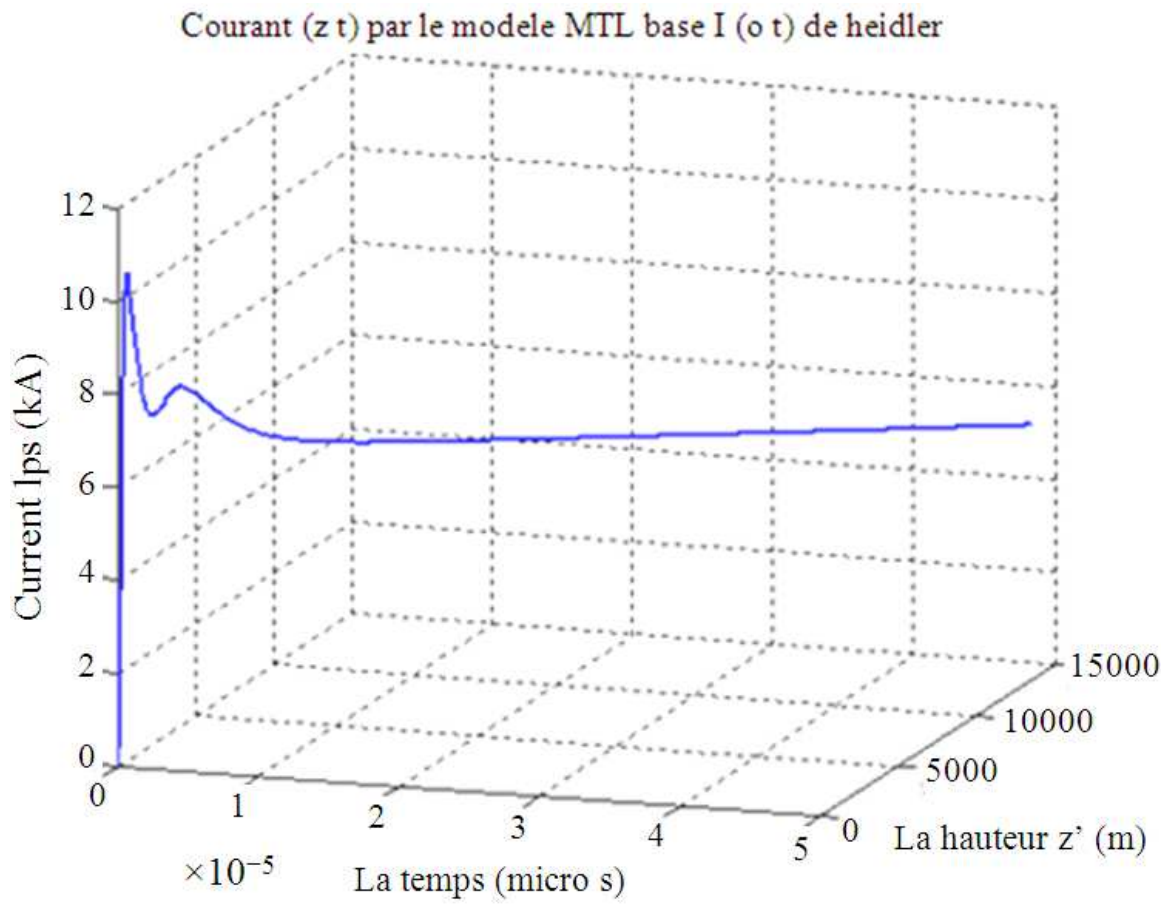

Fig. 6. Current in lightning channel of the Model MTLE with i(0, t) of Heidler case1 (SIMLIGHTNING, 2012) 
Dib Djalel et al. / American Journal of Engineering and Applied Science 6 (4): 369-383, 2013

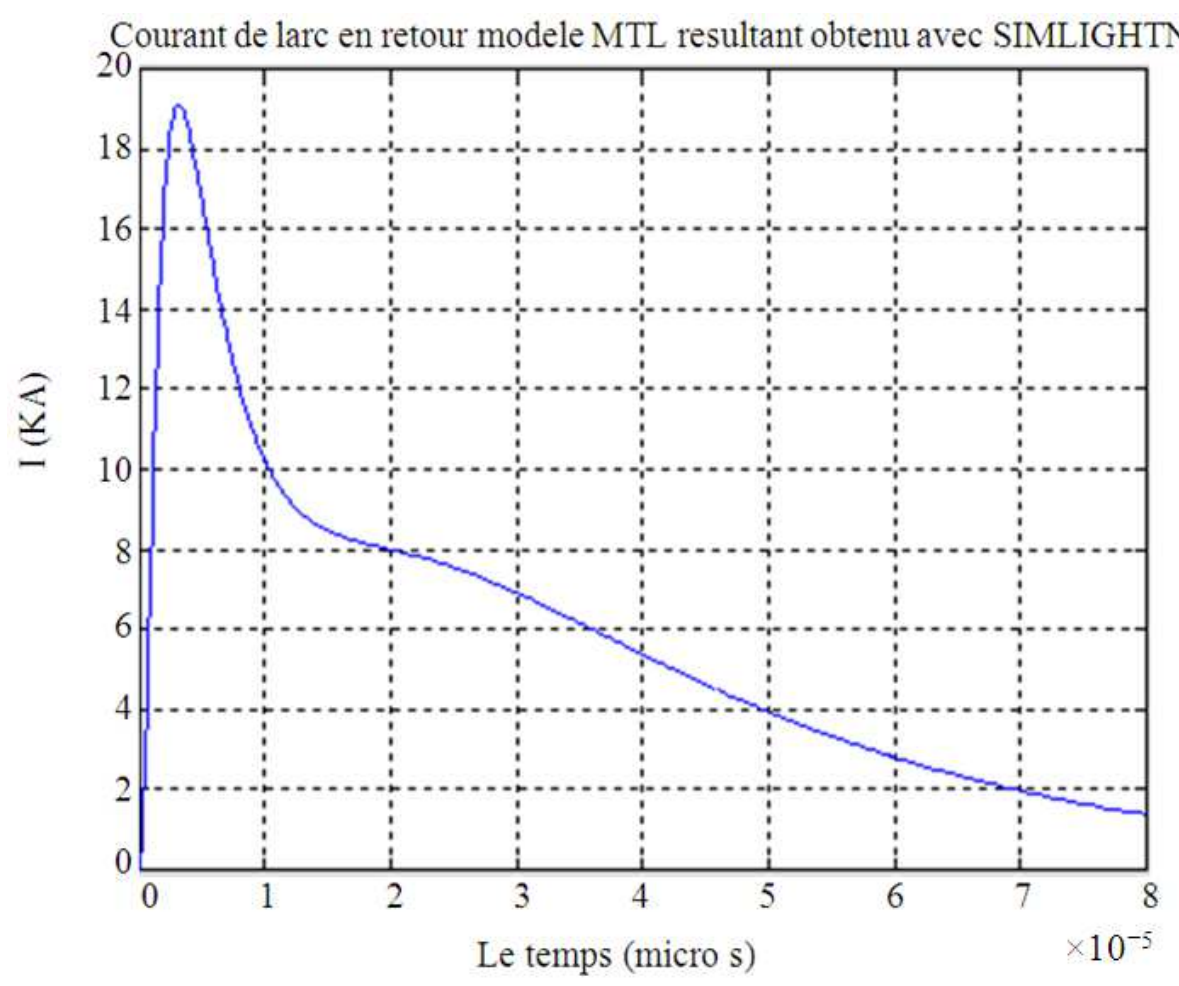

Fig. 7. Current in lightning channel of the Model MTLE with i(0, t) of Heidler case2 (SIMLIGHTNING 2012)

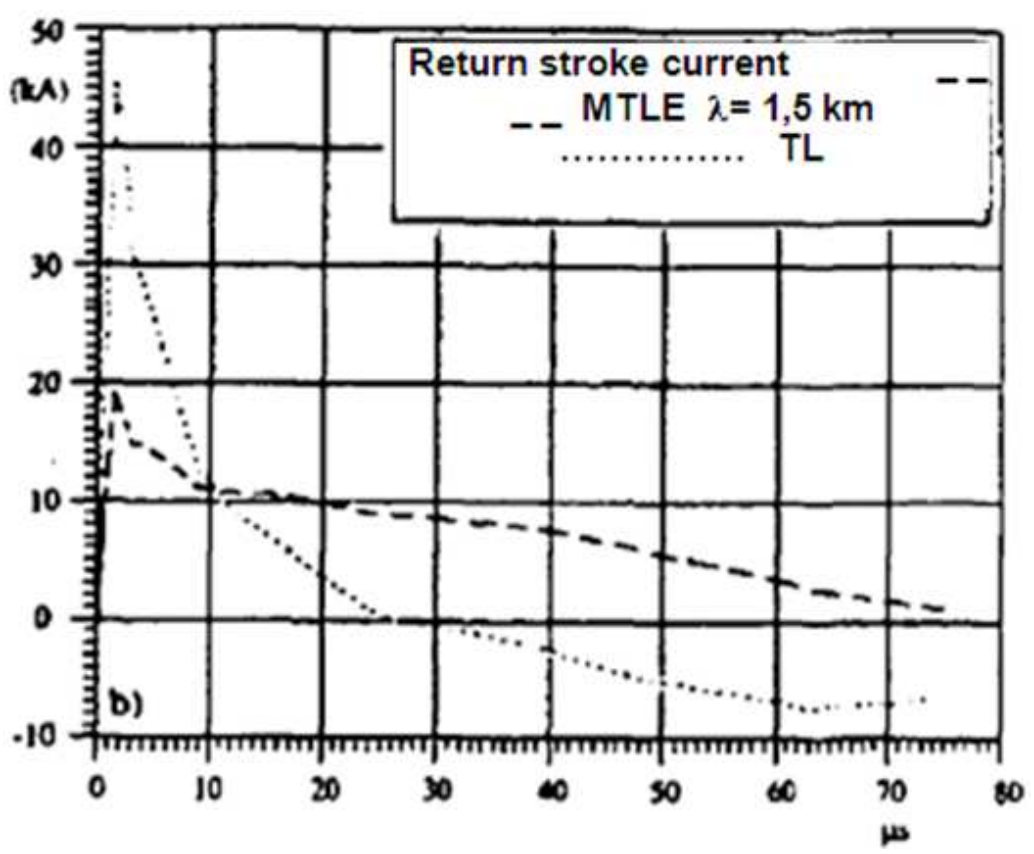

Fig. 8. Return stroke Current in lightning channel, MTLE and TL Model (Cooray, 1994; 1998) 
Dib Djalel et al. / American Journal of Engineering and Applied Science 6 (4): 369-383, 2013

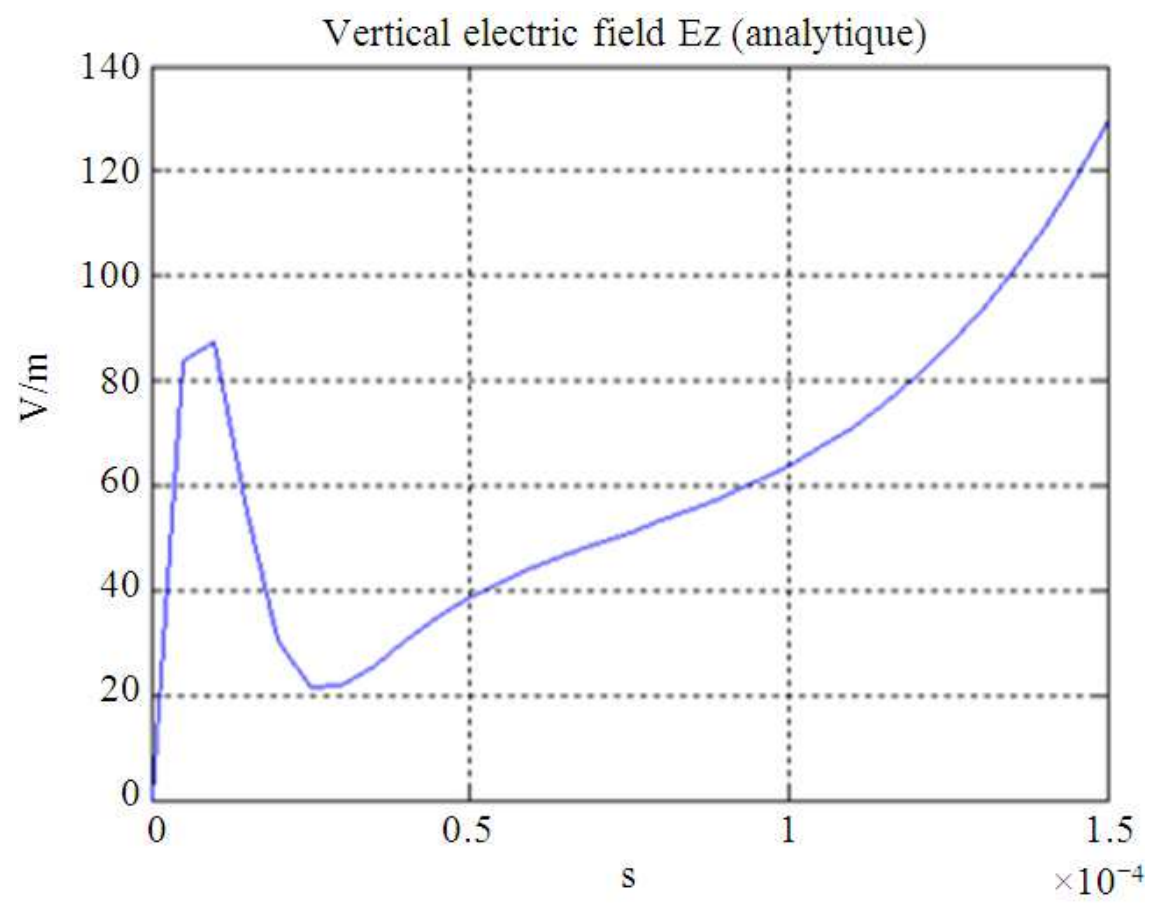

Fig. 9. Vertical Electric fields calculated by the new model (SIMLIGHTNING, 2012) MTLE with i(0, t) of Heidler

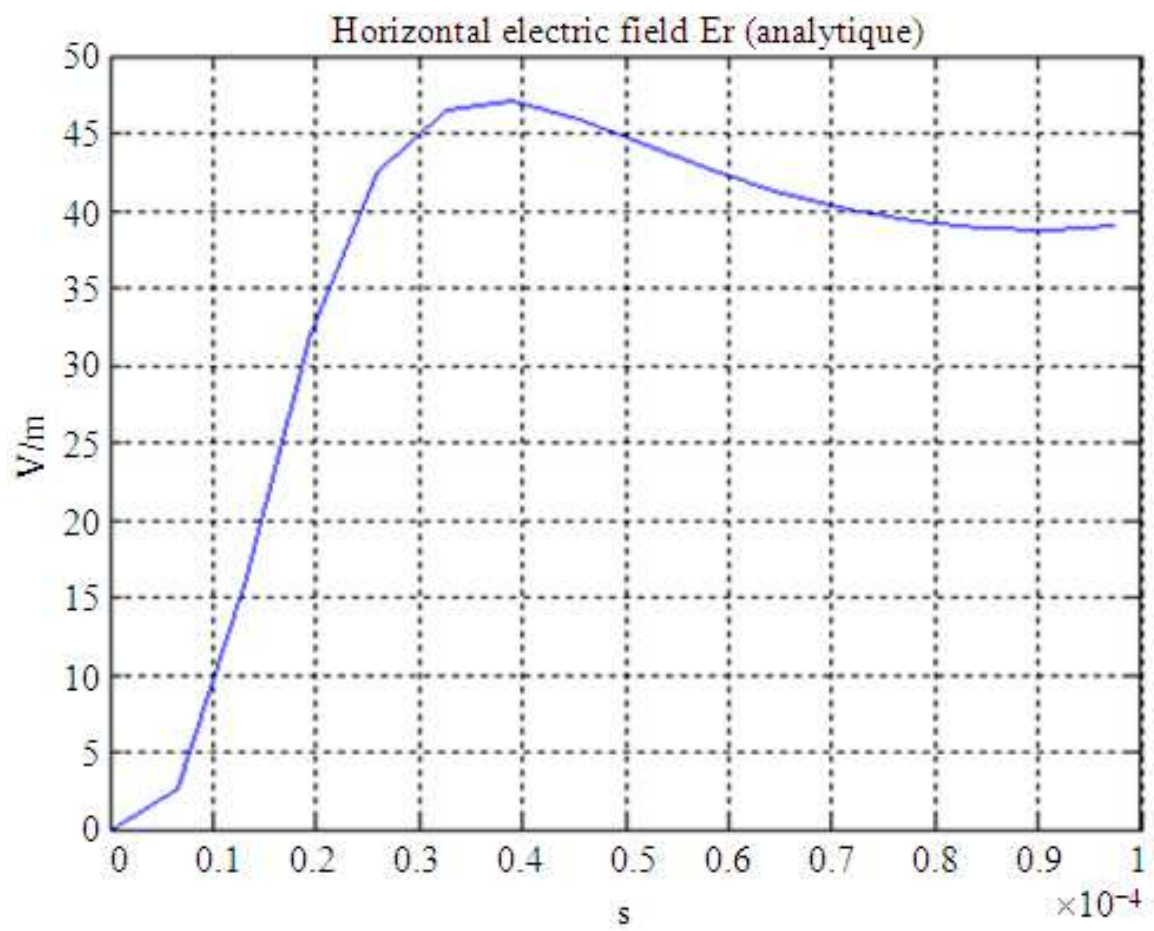

Fig. 10. Horizontal Electric fields calculated by the new model (SIMLIGHTNING, 2012) MTLE with i(0, t) of Heidler 


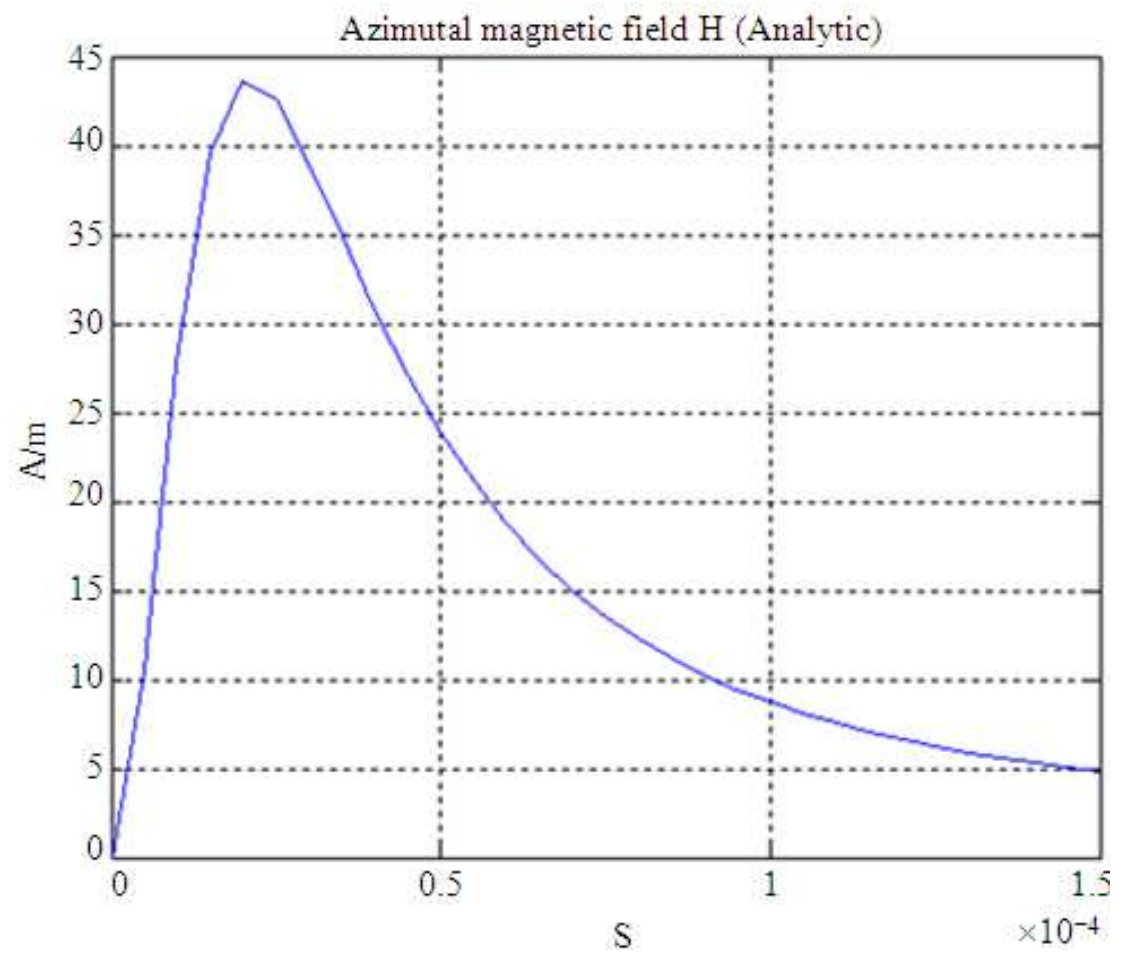

Fig. 11. Azimuth magnetic fields calculated by the new model (SIMLIGHTNING, 2012) MTLE with i(0, t) of Heidler

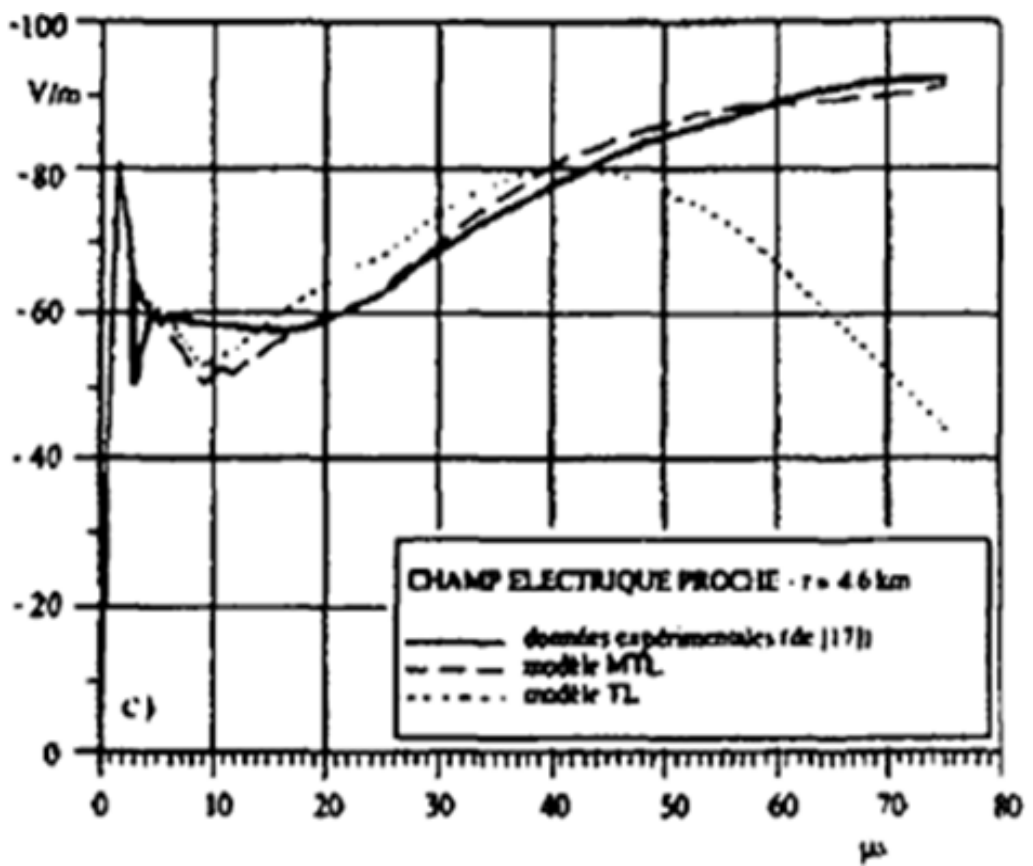

Fig. 12. Vertical Electric fields: Experiment and calculated with MTL and TL model of return stroke Current (Guerrieri et al., 1996; 1998; Rachidi et al., 1992; 1998) 


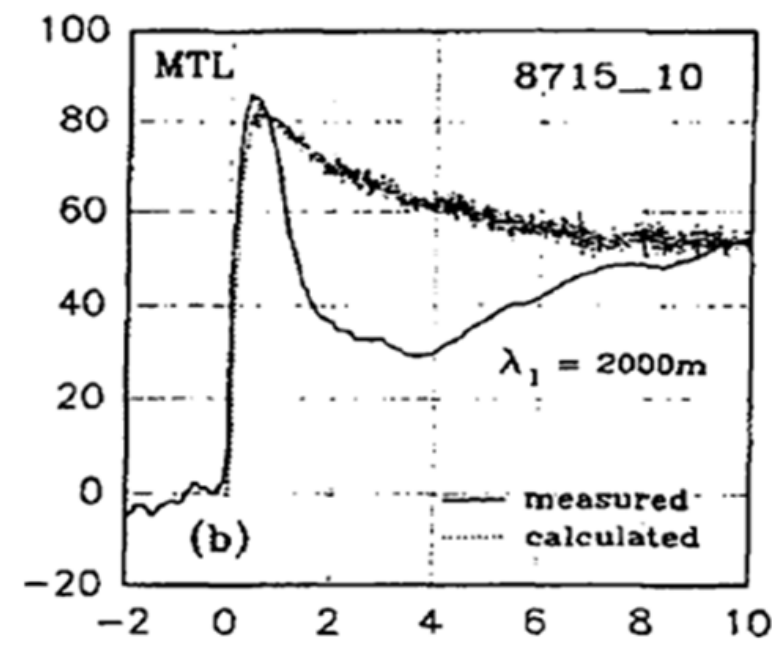

Fig. 13. Horizontal Electric fields: Experiment and calculated with MTL model of return stroke Current (Guerrieri et al., 1996; 1998; Rachidi et al., 1992; 1998)

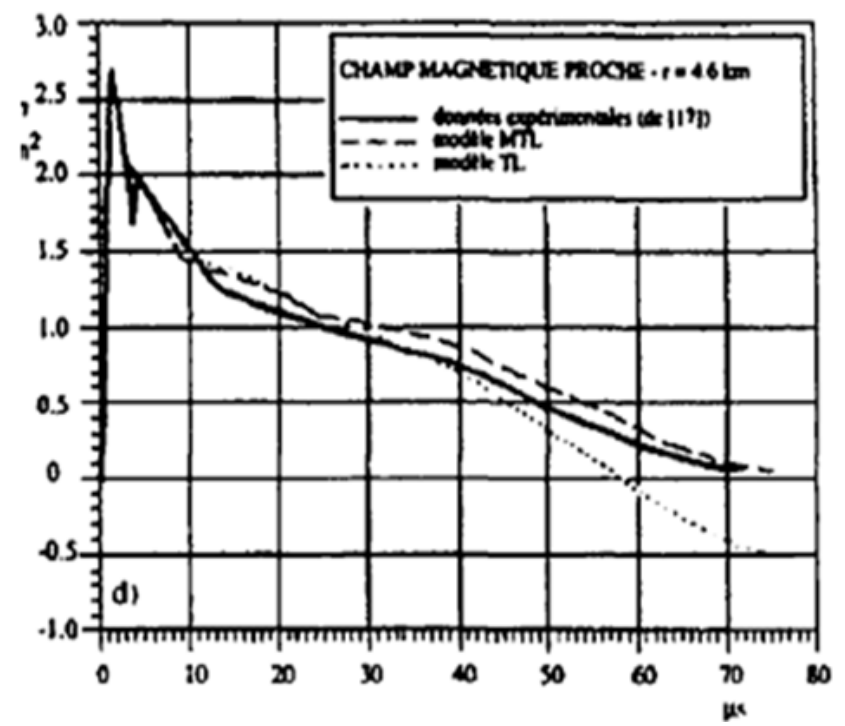

Fig. 14. Magnetic fields: Experiment and calculated with MTL and TL models of return stroke Current (Guerrieri et al., 1996; 1998; Rachidi et al., 1992; 1998)

\section{CONCLUSION}

In this study, we have proposed new equations for electromagnetic fields Radiated by the lightning channel at a point-in-space above-the ground. The analytical development proceeded That We Have Allowed us to models in the time domain by substituting Other variable parameters, Kinds of Space, Geometrical and electrical Such as the current in the lightning channel, Considered the main source of electromagnetic radiation transient.
The theorem of parity symmetric integrals and numerical approaches of Trapez integration and the operator of first order differences, was the various techniques used in the development of new equations. The development of a module named SIMLIGHTNING under Matlab for the simulation of new electromagnetic fields models allows us to have very interesting results of all quantities involved in this development in the lightning current at the canal base, the return current in the lightning canal and the various components of 
electromagnetic fields: Vertical electric field, horizontal electric field and azimuth magnetic field, this is compared with experimental measurements and simulation results published by several authors (Diendorfer and Uman, 1990; Guerrieri et al., 1996; Nucci and Rachidi, 1989; Rachidi et al., 1992; 1998; Dib et al., 2007) and have given a very appreciable similar and affinity.

\section{ACKNOWLEDGEMENT}

I want to thank Prof. Pierre Ezwaikker and Prof F. Rachidi from Labortory of High voltage and EMC of Ecole polytechnic federal of Lausane, Swizerland for their hospitality in their LRE laboratory and and their moral and material support during our stay in 2006.

\section{REFERENCES}

Agrawal, S.P., L.J. Lanzerotti, D. Venkatesan and R.T. Hansen, 1980. Solar polar coronal holes and galactic cosmic ray intensities. J. Geophys. Res., 85: 68456852. DOI: 10.1029/JA085iA12p06845

Cooray, G.V., 2003. On the concepts used in return stroke models applied in engineering practice. IEEE Trans. Electromagn. Compatib., 45: 101-108. DOI: 10.1109/TEMC.2002.808041

Cooray, V., 1994. Calculating lightning-induced overvoltages in power lines. A comparison of two coupling models. IEEE Trans. Electromagn. Compatib., 36: 179-182. DOI: 10.1109/15.305462

Cooray, V., 1998. Predicting the spatial and temporal variation of the electromagnetic fields, currents and speeds of subsequent return strokes. IEEE Trans. Electromagn. Compatib., 40: 427-435. DOI: $10.1109 / 15.736203$

Cooray, V., 2001. Underground electromagnetic fields generated by the return strokes of lightning flashes. IEEE Trans. Electromagn. Compatib., 43: 75-84. DOI: $10.1109 / 15.917942$

Dib, D., A. Haddouche and F. Chemam, 2007. The return-stroke of lightning current, source of electromagnetic fields (study, analysis and modelling). Am. J. Applied Sci., 4: 42-48. DOI: 10.3844/ajassp.2007.42.48

Diendorfer, G. and M.A. Uman, 1990. An improved return stroke model with specified channel-base current. J. Geophys. Res., 95: 13621-13644. DOI: 10.1029/JD095iD09p13621
Guerrieri, S., C.A. Nucci, F. Rachidi and M. Rubinstein, 1998. On the influence of elevated strike objects on directly measured and indirectly estimated lightning currents. IEEE Trans. Power Delivery, 13: 1543-1555. DOI: 10.1109/61.714865

Guerrieri, S., F. Heidler, C.A. Nucci, F. Rachidi and M. Rubinstein, 1996. Extension of two return stroke models to consider the influence of elevated strike objects on the lightning return stroke current and the radiated electromagnetic field: Comparison with experimental results. Proceedings of the International Symposium on Electromagnetic Compatibility, (SEC' 96), Rome, Italy, pp: 701-706.

Heidler, F. and C. Hopf, 1994. Lightning current and lightning electromagnetic impulse considering current reflection at the earth's surface. Proceedings of the 22nd International Conference on Lightning Protection, (ICLP' 94), Budapest, Hungary, pp: 6-6.

Heidler, F., 1985. Traveling current source model for LEMP calculation. Proceedings of the 6th Symposium and Technical Exhibition on Electromagnetic Compatibility, (TEEC' 85), Zurich, Switzerland, pp: 157-162.

Nucci, C.A. and F. Rachidi, 1989. Experimental validation of a modification to the transmission line model for LEMP calculations. Proceedings of the 8th International Symposium on Electromagnetic Compatibility, (EC' 89), Zurich, Switzerland, pp: 6-6.

Rachidi, F., M. Ianoz, C.A. Nucci and C. Mazetti, 1992. Modified transmission line model for LEMP calculations. Effect of the return stroke velocity decreasing and elevated strike object on close fields. Proceedings of the 9th International Conference on Atmospheric Electricity, St. Petersburg, Jun. 15-19, Rusia, pp: 664-667.

Rachidi, F., W. Janischewskyj, A.M. Hussein, C.A. Nucci and S. Guerrieri et al., 1998. Electromagnetic fields radiated by lightning return strokes to high towers. Proceedings of the 24th ICLP, (CLP' 98), Birmingham, UK.

Verbanov, V.D., 2004. Stochastic effects and uncertainties in assessing electromagnetic interactions with control systems. PhD Thesis, Université of Magdeburg, Bulgaria. 